SCIENTIFIC REPORT

\title{
Full thickness eyelid transsection (blepharotomy) for upper eyelid lengthening in lid retraction associated with Graves' disease
}

\author{
C Hintschich, C Haritoglou
}

Br J Ophthalmol 2005;89:413-416. doi: 10.1136/bjo.2004.052852

Aim: To report on a modified technique for upper eyelid lengthening in lid retraction associated with Graves' disease. Methods: A prospective consecutive interventional case series. 41 patients, 38 women and three men, with a mean age of 55 years (range $32-75$ years) were included. Full transsection of the upper eyelid with optional transsection of the lateral horn was performed in 60 lids. A central bridge of conjunctiva was left intact in the pupillary axis. Before and after surgery, the skin crease, palpebral aperture, and scleral show were measured and the contour of the lid margin was assessed. The overall result was graded as "perfect," "acceptable," and "failure."

Results: The mean period review was 6 months (range 2-36 months). Preoperatively, upper eyelid retraction varied from $1 \mathrm{~mm}$ to $7 \mathrm{~mm}$; mean scleral show was $2 \mathrm{~mm}$ in 45 lids, the palpebral aperture was in the desired height (SD $1 \mathrm{~mm}$ ) in 53 of 60 lids (88\%) and was reduced by $3 \mathrm{~mm}$ (mean), the skin crease remained unchanged (SD $1 \mathrm{~mm}$ ) in 44 of 60 lids (73\%). The result was considered "perfect" in 43 and "acceptable" in 14 of 60 lids after one or two surgical approaches. In three lids, the surgery had failed.

Conclusion: In contrast with other surgical techniques the surgical approach presented here is easy to perform and still leads to very satisfying and predictable postoperative results.

$\mathrm{R}$ etraction of the upper eyelid is a very common feature in Graves' disease, often associated with complications such epiphora, foreign body sensation, punctate keratitis or even corneal ulceration and represents a severe cosmetic problem, as retraction of the upper eyelid enhances the proptotic appearance of the patient. Therefore, surgical correction is performed in order to minimise corneal exposure and for cosmetic rehabilitation of the patient.

The challenge for the surgeon is to obtain the most predictable and satisfactory postoperative eyelid contour and height and to minimise the need for further surgery. Multiple approaches have been suggested previously, among them graded Muellerotomy with or without additional recession of the levator aponeurosis, ${ }^{1}$ sclera interposition, ${ }^{23}$ adjustable sutures for graded levator recession ${ }^{45}$ or central aponeurosis disinsertion, ${ }^{6}$ modified levator recession, ${ }^{7}$ and its modification. ${ }^{8}$

We present a new technique of upper eyelid lengthening which is credited to Leo Koornneef from Amsterdam (personal communication).

\section{PATIENTS AND METHODS}

Forty one consecutive patients, 38 female and three male, with a mean age of 55 years (range 32-75 years) were included into this study. Patients with post-traumatic, iatrogenic, neurogenic, or congenital retraction were excluded.

Surgery was performed in 60 eyelids by the first author. Preoperatively, all patients were euthyroid and there was no activity of the disease with all signs and symptoms being stable for at least 6 months. If orbital decompression was indicated, this was performed before lid surgery. All patients were examined 1 day before surgery and at 4 week intervals postoperatively, including visual acuity measurement and a complete slit lamp examination. Palpebral aperture, scleral show, and skin crease were evaluated in millimetres by a ruler and the lid margin contour was assessed. As in most healthy individuals $0.5-1.5 \mathrm{~mm}$ of the cornea are covered by the upper eyelid at the 12 hours position, an eyelid retraction of $1 \mathrm{~mm}$ was diagnosed if the upper eyelid was in line with the limbus. The surgical result was graded according to Mourits et $\mathrm{al}^{8}$ : "perfect" result: $0.5-1.5 \mathrm{~mm}$ of the cornea in the 12 hours position covered by the eyelid, difference of the lid aperture between the left and right eye up to $1 \mathrm{~mm}$, lid contour smooth, skin crease within $7-10 \mathrm{~mm}$ of the lid margin, and the patient being completely satisfied; "acceptable result": upper eyelid within $0.5 \mathrm{~mm}$ of the limbus or not covering more than $2 \mathrm{~mm}$ of the cornea at the 12 hours position, difference of the lid aperture between both eyes up to $2 \mathrm{~mm}$; "failure" was defined as one ore more of these criteria not being fulfilled.

Our surgical approach consisted of the following steps. After disinfection and local anaesthesia with a total of 1 to $1.5 \mathrm{ml}$ bupivacaine $0.5 \%$ with adrenaline (epinephrine) 1:200 000 subcutaneously and subconjunctivally a transcutaneous approach at the level of the skin crease was chosen (fig 1, 2A). Then, all layers except the conjunctiva were transsected (fig 1, 2B). The conjunctiva was then incised in the temporal third of the upper eyelid (fig 2C) and gradually transsected step by step (fig 2E), controlling the achieved height and contour of the lid. If necessary, the lateral horn was transsected, too (fig $2 \mathrm{~F}$ ). In cases of more severe upper lid retraction requiring a subtotal transsection of the conjunctiva, the conjunctiva was also horizontally transsected in the medial third, not exceeding further medially than the upper punctum. Attention was paid to leave a central bridge of conjunctiva intact in the central part of the lid in the pupillary axis (fig $2 \mathrm{G}$ ) This bridge had a minimal horizontal extension of 3-4 $\mathrm{mm}$ in order to maintain a good and natural contour of the eyelid and to avoid a central ptosis. During surgery, the operation table (an electrically driven dentist's chair) was put in an upright position in order to evaluate the position of the upper lids and to achieve the most symmetric result possible. The end point of surgery was determined by the upper eyelid covering the limbus by $0.5-$ $1.5 \mathrm{~mm}$ (depending on the contralateral eyelid and the 

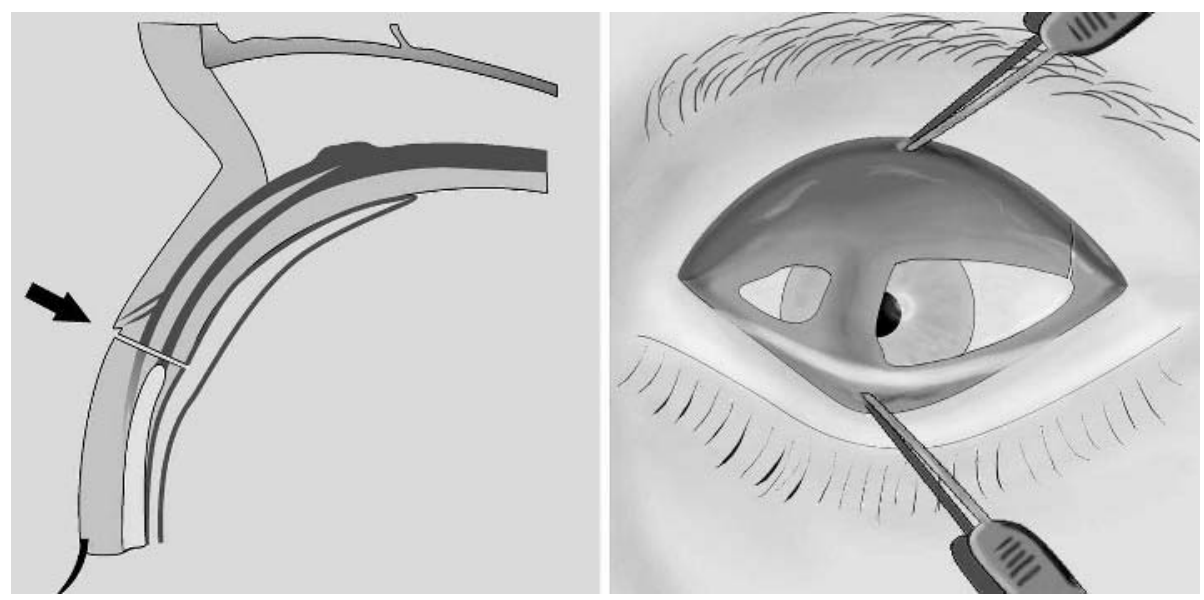

Figure 1 Principle of full thickness eyelid transsection (blepharotomy), sagittal plane (left). Schematic drawing showing central conjuctival bridge and temporal incision of lateral horn (right).
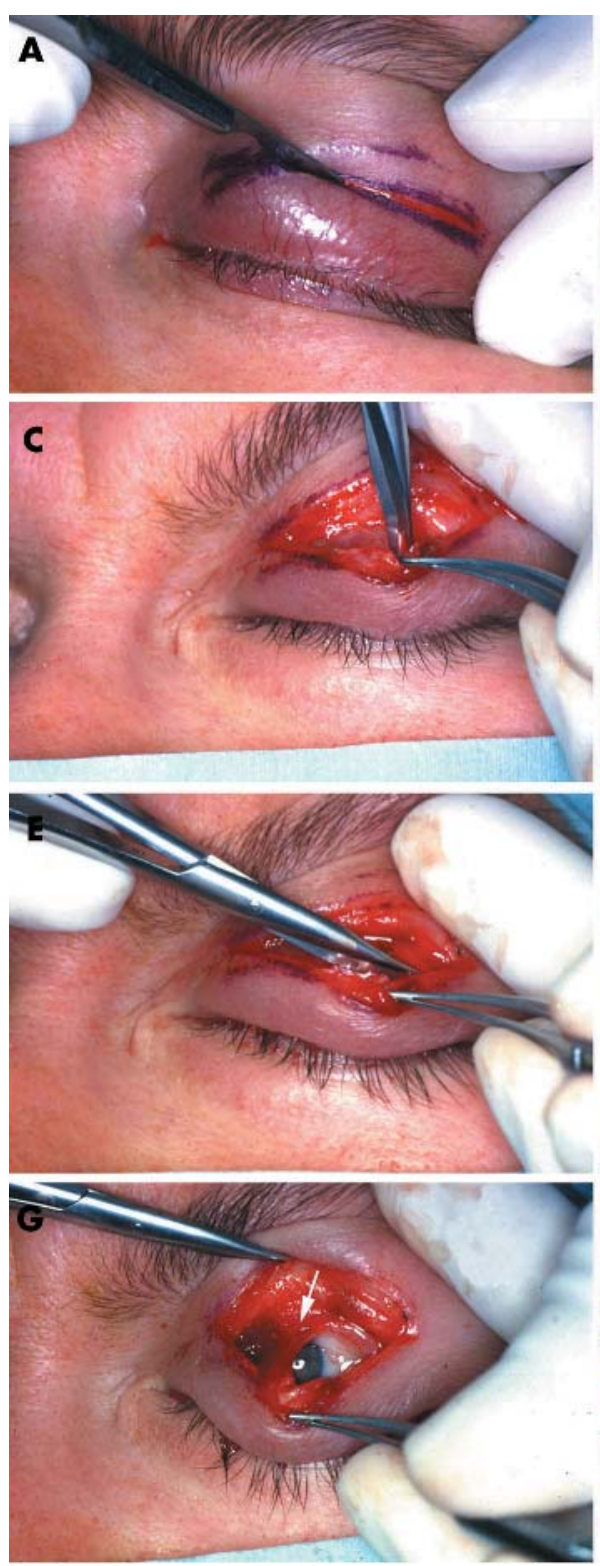
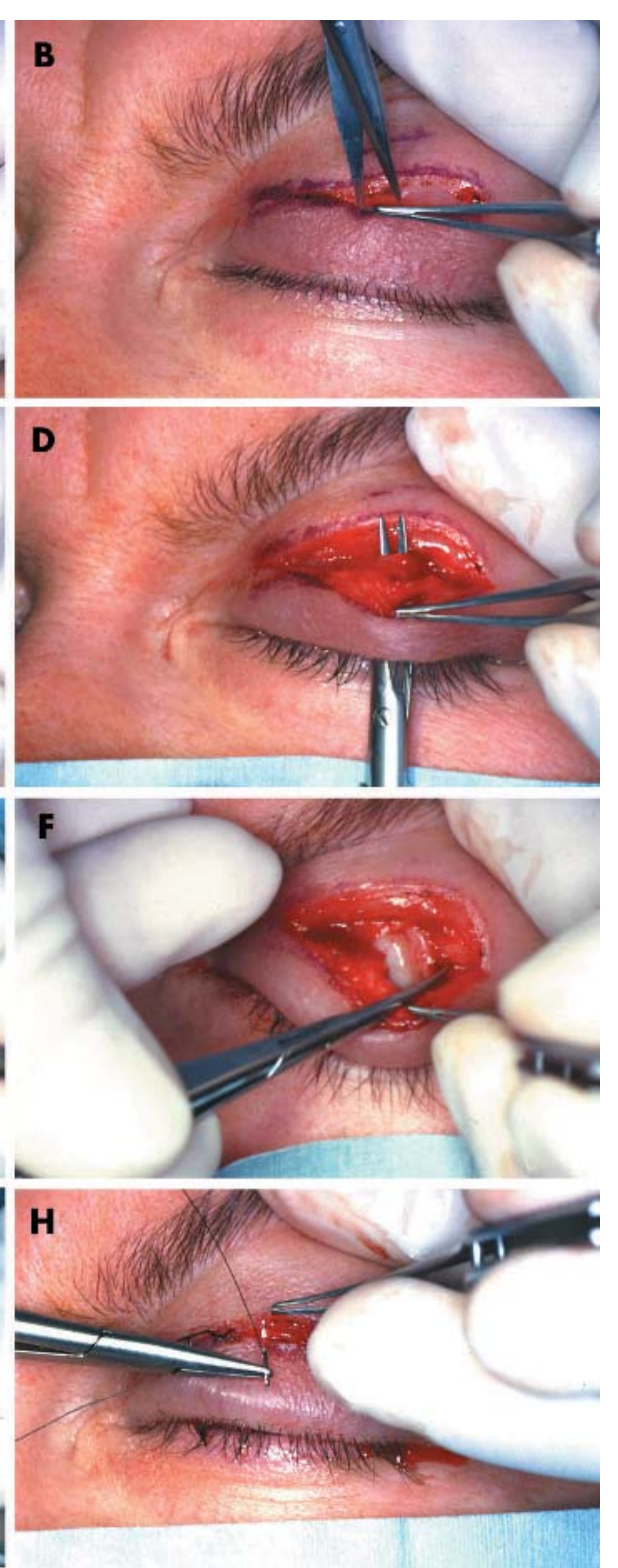

Figure 2 Intraoperative steps of eyelid transsection in a patient with severe upper eyelid retraction (see patient in fig 3A, B). (A) Skin incision in skin crease level. (B) Transsection in a horizontal plane through the orbicularis muscle. (C) Transsection of levator complex and conjunctiva in the lateral third of the upper lid. (D) Branches of scissors indicating full eyelid

transsection. (E) Horizontal transsection of the temporal part of the conjunctiva. (F) Dissection of the lateral horn.

(G) Upper eyelid after subtotal (medial and lateral) horizontal blepharotomy, before wound closure; arrow indicates the remaining bridge of intact

conjunctiva centrally, above the pupil. (H) Skin closure with a 6-0 silk suture with running locked stitches. 
position desired). The wound was then closed by a running locked skin suture with 6-0 black silk (fig $2 \mathrm{H}$ ).

Postoperatively, the patients were encouraged to observe the lid height and contour in a mirror daily. In the case of raising of the upper eyelid during healing they were taught to grab the lid margin and pull the lid down three times a day for up to 4 weeks after surgery and to massage the lid.

\section{RESULTS}

Out of 60 upper eyelids five had undergone lid surgery before. In 35 eyes orbital decompression had been performed to reduce proptosis at least 6 months before. The mean follow up was 6 months (range 2-36 months). Preoperatively, upper eyelid retraction ranged from $1 \mathrm{~mm}$ to $7 \mathrm{~mm}$, a mean scleral show of $2 \mathrm{~mm}$ was noted in 45 lids. Postoperatively, the palpebral aperture was in the desired height (SD $1 \mathrm{~mm}$ ) in $53 / 60$ lids $(88 \%)$, with a mean reduction of the palpebral aperture of $3 \mathrm{~mm}$ (range $0-6 \mathrm{~mm}$ ). A postoperative scleral show was present in three lids, being less than $0.2 \mathrm{~mm}$ in all cases. The skin crease remained unchanged (SD l mm) in 44/ 60 lids (73\%) and increased more than $2 \mathrm{~mm}$ (but not more than $4 \mathrm{~mm}$ ) in five lids. Over all patients, the mean postoperative increase of skin crease height was $0.7 \mathrm{~mm}$.

A postoperative complete or a superficial wound dehiscence was seen in two lids requiring a wound revision in one lid. A second operation with the same technique was performed in six lids because of a recurrence in three or undercorrection in two lids, or according to the patient's demand. Thirty nine of 41 patients were subjectively satisfied with the postoperative result (figs 3, 4). No patient complained of clinical symptoms being suggestive for dry eyes. From the surgeon's point of view, the result was considered "perfect" in 43/60 lids and "acceptable" in 14/60 lids after one or two surgical approaches. In three lids, the surgery had failed according to the criteria described above.

\section{DISCUSSION}

We describe a new technique for upper eyelid lengthening, originally developed by Koornneef. Surgical results of full
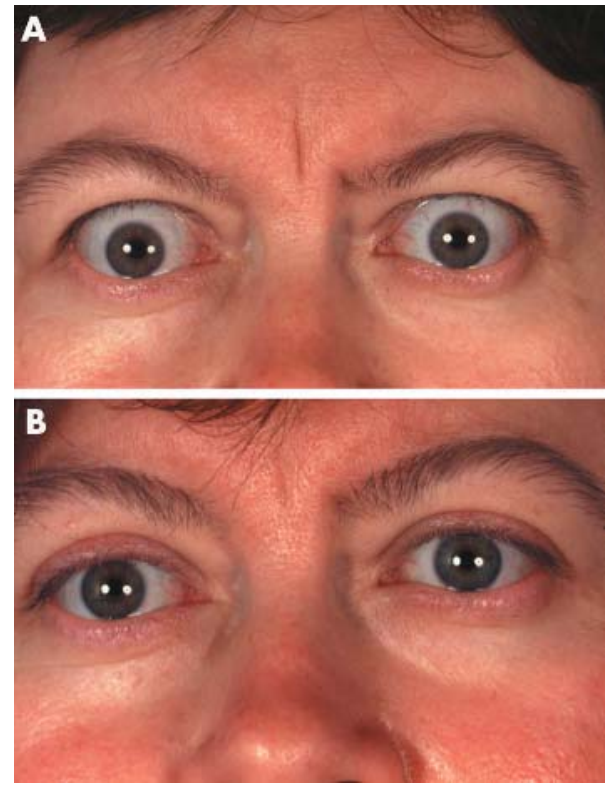

Figure 3 (A) Female patient with severe upper lid retraction, after bilateral orbital decompression. (B) Same patient after bilateral upper lid lengthening using full thickness horizontal blepharotomy (left upper lid lengthening procedure shown in fig 2).
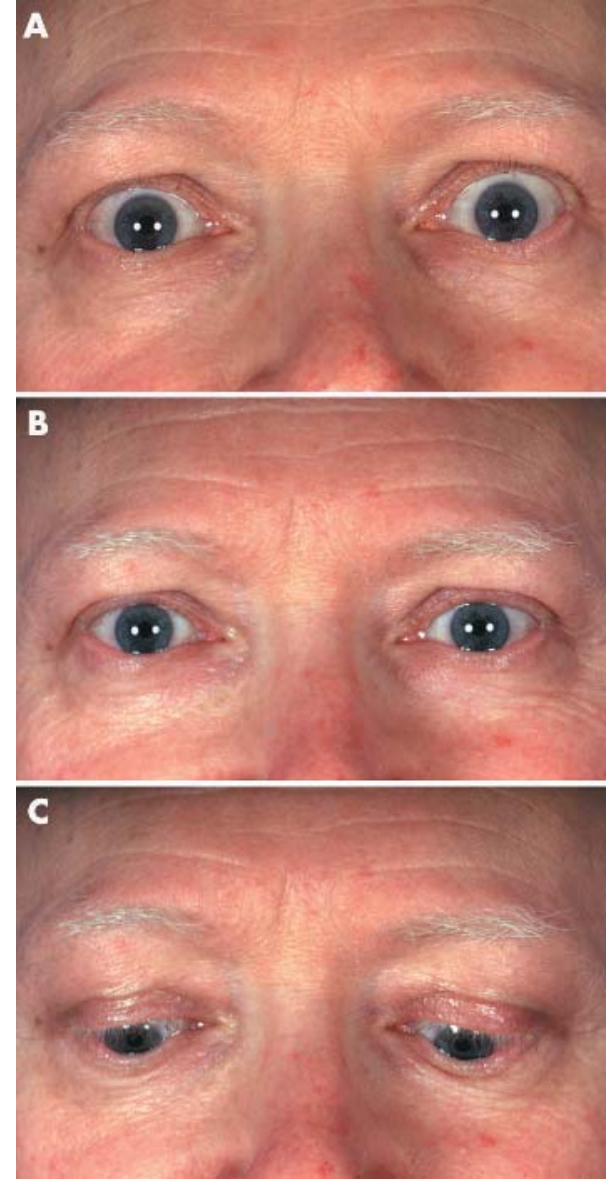

Figure 4 (A) Male patient with marked upper eyelid retraction in Graves' disease. (B) Same patient 6 months after upper eyelid lengthening, primary position. (C) Patient in downgaze, documenting skin crease in normal position.

thickness eyelid transsection according to Kornneef had not been described until recently by Elner, ${ }^{9}{ }^{10}$ confirming our own preliminary data. ${ }^{11}$ However, our technique differs from the procedure described by Elner ${ }^{10}$ in two points: firstly, we always leave a central bridge of conjunctiva of 3-4 mm width above the pupillary axis intact. This guarantees a good and natural postoperative lid contour and avoids central ptosis. A full thickness blepharotomy throughout the whole width of the upper eyelid would result in an unreasonable flat contour, which then would need further adjustment with sutures. Secondly, we preoperatively informed patients about the possibility of postoperative titration of the result by pulling and massaging his lid. This involves them much better in the postoperative care and helped to prevent early recurrences.

The surgical approach presented here leads to very good postoperative results with predictable and satisfactory height and excellent eyelid contour even in severely retracted eyelids and provides two major advantages: firstly, it is simple and does not require great surgical skills or experience compared to other surgical approaches presented in the past $t^{231213}$ and no other sutures than a single running locked skin closure. Secondly, this single technique is applicable for any degree of upper eyelid retraction, as supported by the data presented and by previously published reports. ${ }^{9}$

A few points should be considered in order to achieve best possible results using this technique: 
- Perform a straightforward transsection of skin, orbicularis muscle and levator complex. Don't be afraid to go through the levator aponeurosis. Almost always one needs to cut through the temporal part of the conjunctiva-the amount of transsection is dependent on the intraoperative achievement.

- It is a graded surgery; in more severe cases of lid retraction conjunctival transsection is also carried out in the medial part of the upper eyelid. The obtained amount of upper eyelid lengthening should always be checked with the patient being in an upright position. Therefore, no overcorrection as described in most other methods of lid lengthening procedures, is necessary. Since retraction is usually most pronounced temporally, cutting the lateral horn is almost always mandatory to achieve an adequate height and shape of the eyelid. Any dissection in the vertical plane should be avoided to prevent unnecessary tissue damage leaving anterior adhesions between aponeurotic fibres and skin undisturbed. This might explain why the skin crease remained unaffected in a very high percentage as described above.

This study shows, that graded full thickness eyelid transsection is a reliable and safe method for upper eyelid lengthening with excellent results comparing favourably with the best data in the literature. ${ }^{14713}$ The technique appearsat first glance-rather radical, but is technically straightforward and easy to learn.

\section{ACKNOWLEDGEMENTS}

We thank Leo Koornneef, who was a gifted surgeon, but also an excellent teacher to his fellows, for everything he gave us including the idea of this simplified, but very efficient, approach to upper eyelid lengthening according to his motto "Keep things simple."

\section{Authors' affiliations}

C Hintschich, C Haritoglou, Department of Ophthalmology, LudwigMaximilians-University, Munich, Germany

Financial interest: none.

Correspondence to: Christoph Hintschich, Department of Ophthalmology, Ludwig-Maximilians-University, Munich, Mathildenstrasse 8, D-80335 München, Germany;

christoph.hintschich@med.uni-muenchen.de

Accepted for publication 1 September 2004

\section{REFERENCES}

1 Putterman AM. Graded Müller's muscle excision and levator recession. Am J Ophthalmol 1981;88:507-12.

2 Doxanas MT, Dryden RM. The use of sclera in the treatment of dysthyroid eyelid retraction. Ophthalmology 1981;88:887-94.

3 Mourits MP, Koornneef L. Lid lengthening by sclera interposition for eyelid retraction in Grave's ophthalmopathy. Br J Ophthalmol 1991;75:344-7.

4 Tucker S, Collin R. Repair of upper eyelid retraction: a comparison between adjustable and non-adjustable sutures. Br J Ophthalmol 1995;79:658-660.

5 Woog JL, Harstein ME, Hoenig J. Adjustable suture technique for levator recession. Arch Ophthalmol 1996;114:620-4.

6 Liu D. Surgical correction of upper lid retraction. Ophthalmic Surg 1993;24:323-7.

7 Harvey JT, Corin S, Nixon D, et al. Modified levator aponeurosis recession for upper eyelid retraction in Graves' disease. Ophthalmic Surg 1991:22:313-17.

8 Mourits MP, Sasim I. A single technique to correct various degrees of upper lid retraction in patients with Graves' orbitopathy. Br J Ophthalmol 1999;83:81-4

9 Elner VM, Hassan AS, Frueh BR. Graded full-thickness anterior blepharoomy for upper eyelid retraction. Arch Opthalmol 2004;122:55-60.

10 Elner VM, Hassan AS, Frueh BR. Graded full-thickness anterior blepharotomy for upper eyelid retraction. Trans Am Ophthalmol Soc 2003;101:67-73.

11 Hintschich C, Herzum H. Upper eyelid lengthening-a modified technique. Ophthalmologe 2000;97(Suppl 1):90

12 Mourits MP, et al. Eyelid surgery. In: Prummel MF, ed. Recent developments in Graves' ophthalmopathy. Boston, Dordrecht, London: Kluwer Academic Publishers, 2000:164-5.

13 Older JJ. Surgical treatment of eyelid retraction associated with thyroid eye disease. Ophthalmic Surg 1991;22:318-23. 